\title{
SELF-BOUNDED (A, B)-INVARIANT POLYHEDRA AND CONSTANT REFERENCE TRACKING IN CONSTRAINED LINEAR SYSTEMS
}

\author{
Carlos E. T. Dórea* \\ cetdorea@ufba.br \\ *Universidade Federal da Bahia, Escola Politécnica, Departamento de Engenharia Elétrica \\ Rua Aristides Novis, 2, 40210-630 Salvador, BA, BRAZIL.
}

\begin{abstract}
In this work the concept of self-bounded $(A, B)$-invariant sets is analyzed, as well as its implication in constrained controllability of discrete-time systems subject to state constraints. Self-bounded $(A, B)$-invariant sets are defined and characterized. It is shown that the class of self-bounded sets contained in a given region has an infimum, that is, a selfbounded set which is contained in any set of this class. The infimal set is characterized and a numerical method is presented for its computation in the polyhedral case. These results are then used to analyze the problem of constant reference tracking for state constrained systems. The results are illustrated by a numerical example.
\end{abstract}

KEYWORDS: Linear systems, invariance, geometric approaches, feedback control.

\section{RESUMO}

Neste trabalho, analisa-se o conceito de conjuntos $(A, B)$ invariantes auto-limitados e sua implicação na restrição de controlabilidade de sistemas de tempo discreto sujeitos a restrições lineares nos estados. Conjuntos auto-limitados são definidos e caracterizados. Mostra-se que a classe de conjuntos auto-limitados contidos em uma dada região possui um elemento ínfimo, ou seja, um conjunto auto-limitado que é contido em qualquer outro desta classe. Este conjunto ínfimo é caracterizado e um método numérico é apresentado

\footnotetext{
Artigo submetido em 12/12/02

1a. Revisão em 10/06/03

Aceito sob recomendação do Ed. Assoc. Prof. Liu Hsu
}

para seu cálculo no caso poliédrico. Estes resultados são então usados para se analisar o problema de rastreamento de sinais de referência constantes para sistemas sujeitos a restrições nos estados. Os resultados são ilustrados através de um exemplo numérico.

PALAVRAS-CHAVE: Sistemas lineares, invariância, abordagem geométrica, controle por realimentação.

\section{INTRODUCTION}

Linear systems subject to point-wise-in-time constraints are an object of great interest for both theoreticians and practitioners in control systems, as constraints often arise from physical limitations on input and/or output variables. In particular, the positive invariance approach has been successfully used to solve a large number of problems on constrained dynamical systems. A set in the state space is positively invariant if any trajectory originated from this set does not leave it. An overview of the literature concerning positively invariant sets and their application to the analysis and synthesis of control systems can be found in (Blanchini, 1999).

A key concept of this approach is that of $(A, B)$-invariant (or controlled invariant) sets, which are sets that can be made positively invariant through the choice of a suitable control law (Blanchini, 1994; Dórea and Hennet, 1999) (see also (Bertsekas, 1972; Glover and Schweppe, 1971)). A typical objective in constrained control problems is to force the state trajectory to evolve inside a given region. A possible solution is then to guarantee that the initial state belongs to an $(A, B)$-invariant set contained in the aforementioned region, 
and to apply a control law such that this set is positively invariant. In general, a controlled state trajectory can leave an $(A, B)$-invariant set to reach another one. However, there is a class of $(A, B)$-invariant sets which cannot be exited by trajectories contained in the given region. Such sets characterize a situation of constrained controllability and are known as self-bounded $(A, B)$-invariant sets.

The concept of self-boundedness was first introduced in (Basile and Marro, 1982), but limited to subspaces. In (Dórea and Hennet, 2000), this concept was extended to polyhedral sets. This choice was motivated by the fact that physical limitations inherent to the operation of actual systems very often result in linear constraints on their variables. In this work, the geometrical characterization of self-bounded sets is firstly presented. Necessary and sufficient conditions under which a given polyhedral set is self-bounded are shown. The infimal self-bounded set contained in a given set is then characterized and a numerical algorithm is presented for its computation in the polyhedral case.

The existence of self-bounded sets characterizes a situation of constrained controllability. This is precisely the case when one tries to design a controller so that the system output tracks a reference signal, without violating state constraints. The results on self-bounded polyhedra are then used to determine the set of constant reference signals which can be tracked by the constrained system. The results are illustrated by a numerical example.

Notation: In mathematical expressions, the symbol ":" stands for "such that". 0 represents a null matrix of appropriate dimension. $\operatorname{ker}(M)$ represents null space of matrix $M$. The columns of a matrix $M$ form a generating set of a polyhedral cone $\mathcal{R}$ if and only if there exists a nonnegative vector $\xi$ such that $x=M \xi, \forall x \in \mathcal{R}$. Each column of $M$ is then called a generator of $\mathcal{R}$. A generating set of $\mathcal{R}$ is said to be a minimal generating set if it is defined by the smallest number of generators.

\section{PRELIMINARIES}

Consider the linear, time-invariant, discrete-time system described by:

$$
\begin{aligned}
x(k+1) & =A x(k)+B u(k), \\
y(k) & =C x(k),
\end{aligned}
$$

where $x \in \Re^{n}$ is the state, $u \in \Re^{m}$ is the control input, $y \in \Re^{p}$ is the output and $k$ is a nonnegative integer.

A nonempty closed set $\Omega \subset \Re^{n}$ is said to be positively invariant with respect to a dynamic system $x(k+1)=f(x(k))$ if for all initial state $x(0) \in \Omega$ the state trajectory remains in $\Omega$.
A nonempty closed set $\Omega \subset \Re^{n}$ is said to be $(A, B)$-invariant with respect to system (1) if for all initial state $x(0) \in \Omega$ there exists a control sequence $\{u(k)\}$ such that $x(k) \in \Omega \forall k>0$. Therefore, an $(A, B)$-invariant set is a set which can be made positively invariant through a suitable control action.

It can be shown that, given a convex set $\Omega$, there exists an $(A, B)$-invariant set which contains all those $(A, B)$ invariant sets contained in $\Omega$ :

$\mathcal{C}^{\infty}(\Omega) \triangleq$ supremal $(A, B)$-invariant set contained in $\Omega$.

( $A, B)$-invariance of polyhedra for discrete-time systems was studied in e.g. (Blanchini, 1994; Dórea and Hennet, 1999), where conditions under which a given polyhedron is $(A, B)$-invariant, as well as numerical methods for computation of $\mathcal{C}^{\infty}(\Omega)$ were established.

From now on, the study will be restricted to closed convex sets containing the origin, which are the most relevant for control purposes.

A trajectory of system (1) can be forced to belong to $\Omega$ if and only if the initial state belongs to an $(A, B)$-invariant set contained in $\Omega$, hence in $\mathcal{C}^{\infty}(\Omega)$. Let $\Pi$ be an $(A, B)$-invariant set contained in $\Omega$. In general, for any initial state belonging to $\Pi$, it is possible not only to force the state to remain in $\Pi$ but also to leave it with a trajectory in $\Omega$ and to reach another ( $A, B)$-invariant set contained in $\Omega$. On the contrary, there are $(A, B)$-invariant sets which cannot be exited by means of any trajectory on $\Omega$. Such sets will be studied in next section.

\section{SELF-BOUNDED (A, B)-INVARIANT SETS}

Definition 3.1 An $(A, B)$-invariant set $\Pi$ containing the origin and contained in a set $\Omega$ is said to be self-bounded with respect to $\Omega$ if $x(k) \in \Pi \forall k>0, \forall x(0) \in \Pi$ and for any control sequence $\{u(k)\}$ such that $x(k) \in \Omega \forall k>0$.

In words, $\Pi$ is self-bounded with respect to $\Omega$ if for any $x(0) \in \Pi$, the state vector cannot leave $\Pi$ through trajectories contained in $\Omega$. That is, there is no control sequence $\{u(k)\}$ which drives the state outside $\Pi$ while keeping the state in $\Omega$.

This definition extends to convex sets the concept of selfbounded $(A, B)$-invariant (or controlled invariant) subspaces introduced by Basile and Marro (Basile and Marro, 1982; Basile and Marro, 1992).

Consider now the following convex sets defined in the ex- 
tended space $\Re^{n} \times \Re^{m}$ :

$$
\begin{gathered}
\mathcal{I}(\Pi, \Omega) \triangleq\left\{\left[\begin{array}{l}
x \\
u
\end{array}\right]: x \in \Pi, A x+B u \in \mathcal{C}^{\infty}(\Omega)\right\}, \\
\mathcal{O}(\Pi) \triangleq\left\{\left[\begin{array}{l}
x \\
u
\end{array}\right]: A x+B u \in \Pi\right\} .
\end{gathered}
$$

Theorem 3.1 The convex set $\Pi \subset \Omega$ is self-bounded $(A, B)$ invariant with respect to $\Omega$ if and only if

$$
\mathcal{I}(\Pi, \Omega) \subset \mathcal{O}(\Pi) \text {. }
$$

Proof: Necessity is first proved: Since $\Pi \subset \mathcal{C}^{\infty}(\Omega)$ and $\mathcal{C}^{\infty}(\Omega)$ is $(A, B)$-invariant, then $\forall x(0) \in \Pi$, there always exists a control sequence $\{u\}\left(\mathcal{C}^{\infty}(\Omega)\right)=$ $\{u(0), u(1), u(2), \ldots\}$ such that $x(k) \in \mathcal{C}^{\infty}(\Omega) \forall k>0$. Suppose now that there exists $\left[\begin{array}{l}x_{n} \\ u_{n}\end{array}\right] \in \mathcal{I}(\Pi, \Omega)$ such that $A x_{n}+B u_{n} \notin \Pi$. Then, it is clear that for $x(0)=x_{n}$ there exists a trajectory of the state completely contained in $\Omega$, but which leaves $\Pi$, contradicting thereby the assumption that $\Pi$ is self-bounded.

Sufficiency comes from the fact that if (4) is verified, then any trajectory starting from $\Pi$ and completely contained in $\mathcal{C}^{\infty}(\Omega)$ (hence, in $\Omega$ ) will not leave $\Pi$.

Corollary 3.1 Let $\{u(k)\}\left(\mathcal{C}^{\infty}(\Omega)\right)$ be any control sequence $\{u(0), u(1), u(2), \ldots\}$ such that, $x(k) \in \mathcal{C}^{\infty}(\Omega), \forall x(0) \in$ $\mathcal{C}^{\infty}(\Omega), \forall k>0$. Then, any $(A, B)$-invariant set $\Pi$, selfbounded with respect to $\Omega$, is such that $x(k) \in \Pi, \forall x(0) \in$ П, $\forall k>0, \forall\{u(k)\}\left(\mathcal{C}^{\infty}(\Omega)\right)$.

Proof: It suffices to notice that any vector $\left[\begin{array}{l}x(k) \\ u(k)\end{array}\right]$, corresponding to the state and the control in time $k$, where $x(0) \in \Pi, x(k) \in \mathcal{C}^{\infty}(\Omega)$ and $u(k)$ is in $\{u(k)\}\left(\mathcal{C}^{\infty}(\Omega)\right)$, is such that $\left[\begin{array}{l}x(k) \\ u(k)\end{array}\right] \in \mathcal{I}(\Pi, \Omega)$. Therefore, if condition (4) of self-boundedness is verified, then $x(k) \in \Pi \forall k \geq 0$.

This corollary states that any control law for which $\mathcal{C}^{\infty}(\Omega)$ is positively invariant is such that any self-bounded set contained in $\Omega$ is also positively invariant under this law.

The study will now be specialized to convex polyhedra containing the origin, represented by sets of linear inequalities:

$$
\begin{array}{r}
\Omega=R[W, \zeta]=\{x: W x \leq \zeta\}, \quad \zeta \geq 0 \\
\Pi=R[G, \rho]=\{x: G x \leq \rho\}, \quad \rho \geq 0 .
\end{array}
$$

where $W$ and $G$ are matrices and $\zeta$ and $\rho$ are vectors of appropriate dimensions. Let also the supremal $(A, B)$-invariant set contained in $\Omega$ be a convex polyhedron represented by:

$$
\mathcal{C}^{\infty}(\Omega)=R[V, \nu]=\{x: V x \leq \nu\}, \nu \geq 0 .
$$

The hypothesis above is not always verified. $\mathcal{C}^{\infty}(\Omega)$ may be defined by an infinite number of linear inequalities. In practice, however, this is not a serious drawback: as shown in (Blanchini, 1994), under mild assumptions, $\mathcal{C}^{\infty}(\Omega)$ can be arbitrarily approximated by an $(A, B)$-invariant polyhedron $R[V, \nu]$.

Theorem 3.2 The convex polyhedron $R[G, \rho] \subset R[W, \zeta]$ is self-bounded $(A, B)$-invariant with respect to $R[W, \zeta]$ if and only if there exist matrices $L$ and $M$ such that:

$$
\begin{gathered}
L G+M V A=G A, \\
M V B=G B, \\
L \rho+M \nu \leq \rho, \\
L \geq 0, M \geq 0 .
\end{gathered}
$$

Proof: See (Dórea and Hennet, 2000).

The matrix relations in (5) are linear. Self-boundedness of a polyhedron can therefore be checked through the resolution of linear programming problems as for the classical positive invariance relations (see e.g. (Hennet, 1995)).

\section{THE INFIMAL SELF-BOUNDED SET}

The family of $(A, B)$-invariant sets contained in a given convex set, say $\Omega$, is an upper semilattice with respect to the operation "convex hull of the union". This property guarantees the existence, in this family of the supremal set $\mathcal{C}^{\infty}(\Omega)$.

The most outstanding property of the family of self-bounded $(A, B)$-invariant sets contained in a given set is to be a lattice instead of a semilattice. Hence it admits both a supremum $\left(\mathcal{C}^{\infty}(\Omega)\right)$ and an infimum. The existence of an infimum is guaranteed by the following property:

Property 4.1 The family of all self-bounded ( $A, B)$ invariant sets contained in a convex set $\Omega$ is closed under intersection.

Proof: It follows immediately from the Definition 3.1. Let $\Pi_{1}$ and $\Pi_{2}$ be two self-bounded $(A, B)$-invariant sets contained in $\Omega$. Then it is clear that any trajectory starting from $x(0) \in \Pi_{1} \cap \Pi_{2}$ and contained in $\Omega$ can leave neither $\Pi_{1}$ nor $\Pi_{2}$, hence $\Pi_{1} \cap \Pi_{2}$. Therefore, $\Pi_{1} \cap \Pi_{2}$ is self-bounded $(A, B)$-invariant. 
Since $\Omega$ is closed by assumption, this property guarantees the existence, in the family of self-bounded $(A, B)$-invariant sets contained in $\Omega$, of an infimal element (an element which is contained in all the other elements):

$$
\mathcal{C}_{\infty}(\Omega) \triangleq \begin{gathered}
\text { infimal self-bounded }(A, B) \text {-invariant set } \\
\text { contained in } \Omega \text {. }
\end{gathered}
$$

$\mathcal{C}_{\infty}(\Omega)$ is the set defined by the intersection of all selfbounded $(A, B)$-invariant sets in $\Omega$. It should be noticed that $\mathcal{C}_{\infty}(\Omega)$ cannot be empty as it was assumed that the origin belongs to any self-bounded set.

Theorem 4.1 Consider the following sequence of sets:

$$
\begin{aligned}
\mathcal{C}_{0}= & \{0\} \\
\mathcal{C}_{i+1}= & \left\{x \in \mathcal{C}^{\infty}(\Omega): \exists x_{i} \in \mathcal{C}_{i}, u_{i}:\right. \\
& \left.x=A x_{i}+B u_{i}\right\} .
\end{aligned}
$$

The infimal self-bounded $(A, B)$-invariant set contained in $\Omega$ is given by: $\mathcal{C}_{\infty}(\Omega)=\lim _{i \rightarrow \infty} \mathcal{C}_{i}(i=0,1,2, \ldots)$.

Proof: First we prove, by induction, that $\mathcal{C}_{i} \subset \mathcal{C}_{i+1} \forall i=$ $0,1,2, \ldots$ Suppose $\mathcal{C}_{i-1} \subset \mathcal{C}_{i}$. Then, it is clear that any $x \in$ $\mathcal{C}^{\infty}(\Omega)$ such that there exist $x_{i} \in \mathcal{C}_{i-1}$ and $u_{i}$ for which $x=A x_{i}+B u_{i}$ belongs to $\mathcal{C}_{i+1}$. In other words, any $x \in \mathcal{C}_{i}$ belongs to $\mathcal{C}_{i+1}$. Therefore, if $\mathcal{C}_{i-1} \subset \mathcal{C}_{i}$ then $\mathcal{C}_{i} \subset \mathcal{C}_{i+1}$. Since clearly $\mathcal{C}_{0} \subset \mathcal{C}_{1}$, then, by induction, $\mathcal{C}_{i} \subset \mathcal{C}_{i+1} \forall i=$ $0,1,2, \ldots$

Consider now an admissible trajectory starting from $x(0) \in$ $\mathcal{C}_{\infty}(\Omega)$. A trajectory will be said to be admissible if it does not leave $\Omega$ (hence $\left.\mathcal{C}^{\infty}(\Omega)\right) \forall k>0$. Since $\mathcal{C}_{i} \subset \mathcal{C}_{\infty}(\Omega)$ $\forall i=0,1,2, \ldots$ then $x(0) \in \mathcal{C}_{i}$ for some $i$. Hence, $x(1) \in$ $\mathcal{C}_{i+1} \subset \mathcal{C}_{\infty}(\Omega)$. Therefore, any admissible trajectory is such that $x(k) \in \mathcal{C}_{\infty}(\Omega) \forall k>0$. This proves that $\mathcal{C}_{\infty}(\Omega)$ is self-bounded $(A, B)$-invariant.

Let now $\mathcal{C} \subset \Omega$ be an arbitrary self-bounded $(A, B)$-invariant set, and suppose $\mathcal{C}_{i} \subset \mathcal{C}$ for some $i$. Then, $A x_{i}+B u_{i} \in \mathcal{C}$ $\forall x_{i} \in \mathcal{C}, u_{i}$ such that $A x_{i}+B u_{i} \in \mathcal{C}^{\infty}(\Omega)$. Therefore, $x=$ $A x_{i}+B u_{i} \in \mathcal{C} \forall x_{i} \in \mathcal{C}_{i}, u_{i}$ such that $A x_{i}+B u_{i} \in \mathcal{C}^{\infty}(\Omega)$. Hence, any $x \in \mathcal{C}_{i+1}$ also belongs to $\mathcal{C}$. Therefore, $\mathcal{C}_{i} \subset \mathcal{C}$ $\forall i$ hence $\mathcal{C}_{\infty}(\Omega) \subset \mathcal{C}$. This proves that $\mathcal{C}_{\infty}(\Omega)$ is infimal.

$\mathcal{C}_{i}$ is the set of states which can be reached from the origin in $i$ steps by means of a control sequence such that $x(k) \in$ $\mathcal{C}^{\infty}(\Omega) \forall k \geq 0$. Therefore, one can conclude that $\mathcal{C}_{\infty}(\Omega)$ is the set of reachable states from the origin inside $\mathcal{C}^{\infty}(\Omega)$. It can be also noticed that $\mathcal{C}_{i+1}$ is the projection of $\mathcal{I}\left(\mathcal{C}_{i}, \Omega\right)$ onto the state space $\Re^{n}$.

The focus is now turned towards the computation of $\mathcal{C}_{\infty}(\Omega)$ when $\Omega$ is a polyhedral set represented by $R[W, \zeta]$ and $\mathcal{C}^{\infty}(\Omega)=R[V, \nu]$. Let the set $\mathcal{C}_{i}$ be given by: $\mathcal{C}_{i}=\{x$ :
$\left.G_{i} x \leq \rho_{i}\right\} .>$ From (6), the set $\mathcal{C}_{i+1}$ is then given by:

$$
\begin{aligned}
\mathcal{C}_{i+1}= & \left\{x: \exists x_{i}, u_{i}: x-A x_{i}-B u_{i}=0,\right. \\
& \left.G_{i} x_{i} \leq \rho_{i}, V x \leq \nu\right\}
\end{aligned}
$$

The relations defining $\mathcal{C}_{i+1}$ can be written in the following matrix form:

$$
\left[\begin{array}{c}
I \\
-I \\
0 \\
V
\end{array}\right] x+\left[\begin{array}{cc}
-A & -B \\
A & B \\
G_{i} & 0 \\
0 & 0
\end{array}\right]\left[\begin{array}{l}
x_{i} \\
u_{i}
\end{array}\right] \leq\left[\begin{array}{c}
0 \\
0 \\
\rho_{i} \\
\nu
\end{array}\right]
$$

The dependency on $x_{i}$ and $u_{i}$ in the definition of $\mathcal{C}_{i+1}$ can be eliminated as follows. Let the rows of matrix $T=$ $\left[T_{1}^{+} T_{1}^{-} T_{2}\right]$ form a minimal generating set of the polyhedral cone defined by the vectors $w=\left[\begin{array}{ll}w_{1}^{+} & w_{1}^{-} w_{2}\end{array}\right]$, with $w_{1}^{+}, w_{1}^{-}, w_{2} \geq 0$, such that: $\left[\begin{array}{lll}w_{1}^{+} & w_{1}^{-} & w_{2}\end{array}\right]\left[\begin{array}{cc}-A & -B \\ A & B \\ G_{i} & 0\end{array}\right]=$ $\left[\begin{array}{ll}0 & 0\end{array}\right]$. Then, from application of Farkas' Lemma (Schrijver, 1987; Dórea and Hennet, 1999), $\exists x_{i}, u_{i}$ such that the first three inequalities in (7) are verified if and only if $\left[T_{1}^{+} T_{1}^{-} T_{2}\right]\left(\left[\begin{array}{c}I \\ -I \\ 0\end{array}\right] x-\left[\begin{array}{c}0 \\ 0 \\ \rho_{i}\end{array}\right]\right) \leq 0$ Therefore, the set $\mathcal{C}_{i+1}$ is given by: $\mathcal{C}_{i+1}=$ $\left\{x:\left[\begin{array}{c}T_{1}^{+}-T_{1}^{-} \\ V\end{array}\right] x \leq\left[\begin{array}{c}T_{2} \rho_{i} \\ \nu\end{array}\right]\right\}$.

As shown in (Keerthi and Gilbert, 1987), it is possible to compute matrix $T$ by means of Fourier-Motzkin elimination technique (Schrijver, 1987).

After suppression of redundant constraints, the polyhedral set $\mathcal{C}_{i+1}$ can be written in the form: $\mathcal{C}_{i+1}=\left\{x: G_{i+1} x \leq\right.$ $\left.\rho_{i+1}\right\}$. Algorithm (6) is then run until $\mathcal{C}_{i+1}=\mathcal{C}_{i}$ up to a given accuracy. Since all the sets in question are polyhedra, this test can be performed via linear programming through application of Farkas' Lemma (Hennet, 1995).

If $\Omega=R[W, \zeta]$ is an unbounded symmetrical polyhedron, then the set $\mathcal{C}_{\infty}(\Omega)$ can have infinite directions as well. In this case, the preceding procedure is not able to compute $\mathcal{C}_{\infty}(\Omega)$. One is then led to decompose its computation by "subtracting" from $\Omega$ the minimal self-bounded $(A, B)$ invariant subspace contained in $\operatorname{ker}(W)$ and applying the algorithm above to a polyhedron defined on a reduced order space (see (Dórea and Hennet, 1999) for further details).

\section{CONSTANT REFERENCE TRACKING UNDER STATE CONSTRAINTS}

The goal now is to determine conditions under which it is possible to compute a control law $u(k), k=0,1, \cdots$ such 
that the output $y(k)$ tracks a constant reference signal $r$, respecting at the same time the state constraints, that is, for all $x(0) \in \Omega:\left\{\begin{array}{l}\lim _{k \rightarrow \infty} y(k)=r, \\ x(k) \in \Omega \forall k \geq 0 .\end{array}\right.$

The first specification can be achieved only if $r=C \bar{x}$, where $\bar{x}$ is an equilibrium point, that is, there exists a $\bar{u}$ such that:

$$
\bar{x}=A \bar{x}+B \bar{u} .
$$

Let $N_{B}$ be a matrix whose rows form a basis for the left null space of matrix $B$. The set of equilibrium points is then given by:

$$
\left\{\bar{x}: N_{B}(I-A) \bar{x}=0\right\} .
$$

Also, it is clear from the development of the preceding sections that the second specifications can be achieved only if:

$$
\bar{x} \in \mathcal{C}_{\infty}(\Omega) .
$$

Indeed, if $\bar{x} \notin \mathcal{C}^{\infty}(\Omega)$, then, for some $x(0) \in \Omega$, any control law such that $\lim _{k \rightarrow \infty} x(k)=\bar{x}$ would violate the constraint $x(k) \in \Omega$. Moreover, if $\bar{x} \in \mathcal{C}^{\infty}(\Omega)$ but $\bar{x} \notin \mathcal{C}_{\infty}(\Omega)$, then, due to the self-boundedness of $\mathcal{C}_{\infty}(\Omega)$, for any $x(0) \in$ $\mathcal{C}_{\infty}(\Omega) \subset \Omega, \bar{x}$ is not reachable from trajectories which respect the state constraints.

Therefore, the set of trackable constant references is given by:

$$
\mathcal{Y}_{R}=\left\{r: r=C \bar{x}, \text { with } \begin{array}{l}
N_{B}(I-A) \bar{x}=0 \\
\bar{x} \in \mathcal{C}_{\infty}(\Omega)
\end{array}\right\} .
$$

Consider now the polyhedral case, for which:

$$
\begin{aligned}
& \Omega=R[W, \zeta]=\{x: W x \leq \zeta\}, \quad \zeta \geq 0, \\
& \mathcal{C}_{\infty}(\Omega)=R[G, \rho]=\{x: G x \leq \rho\} .
\end{aligned}
$$

In this case:

$$
\begin{aligned}
\mathcal{Y}_{R}= & \{r: \exists \bar{x}: r-C \bar{x}=0, \\
& \left.N_{B}(I-A) \bar{x}=0, G x \leq \rho\right\} .
\end{aligned}
$$

The relations defining $\mathcal{Y}_{R}$ can be written in the following matrix form:

$$
\left[\begin{array}{c}
I \\
-I \\
0 \\
0 \\
0
\end{array}\right] r+\left[\begin{array}{c}
-C \\
C \\
N_{B}(I-A) \\
-N_{B}(I-A) \\
G
\end{array}\right] \bar{x} \leq\left[\begin{array}{l}
0 \\
0 \\
0 \\
0 \\
\rho
\end{array}\right] .
$$

Let the rows of matrix $U=\left[U_{1}^{+} U_{1}^{-} U_{2}^{+} U_{2} U_{3}\right]$ form a minimal generating set of the polyhedral cone defined by the vectors $u=\left[\begin{array}{lllll}u_{1}^{+} & u_{1}^{-} & u_{2}^{+} & u_{2}^{-} & u_{3}\end{array}\right]$, with $u_{1}^{+}, u_{1}^{-}, u_{2}^{+}, u_{2}^{-}, u_{3} \geq 0, \quad$ such that: $\left[u_{1}+u_{1}-u_{2}+u_{2}-u_{3}\right]\left[\begin{array}{c}, u_{3} \\ -C \\ C \\ N_{B}(I-A) \\ -N_{B}(I-A) \\ G\end{array}\right] \bar{x}=0$.

Then, from application of Farkas' Lemma, $\exists \bar{x}$ such that (8) is verified if and only if:

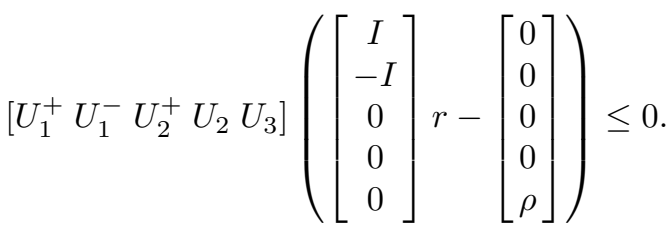

Therefore, the set $\mathcal{Y}_{R}$ is the polyhedron given by:

$$
\mathcal{Y}_{R}=\left\{r:\left(U_{1}^{+}-U_{1}^{-}\right) r \leq U_{3} \rho\right\} .
$$

The focus is now turned towards the computation of a control law which forces the system to track a given reference $r \in$ $\mathcal{Y}_{R}$.

It is well known that the trajectory of a system can be confined in an $(A, B)$-invariant polyhedron through a piecewise linear state feedback control law $u(k)=\phi(x(k))$ (Blanchini, 1994; Dórea and Hennet, 1999). If the polyhedron is contractive, then under this control law $x(k) \rightarrow 0$ when $k \rightarrow \infty$.

In (Blanchini and Miani, 2000) it is shown that a control law which achieves asymptotic tracking can be derived from $\phi(x)$ by applying a translation to the state and control variables, so that the new variables are given by: $\hat{x}=x-\bar{x}$, $\hat{u}=u-\bar{u}$. Clearly, a control law such that $\hat{x}(k) \rightarrow 0$ achieves $x(k) \rightarrow \bar{x}$ (thus $y(k) \rightarrow r$ ) when $k \rightarrow \infty$.

\section{NUMERICAL EXAMPLE}

Consider the system (1) for which:

$$
\begin{gathered}
A=\left[\begin{array}{cc}
-0.8 & 0.2 \\
0.5 & -0.9
\end{array}\right], B=\left[\begin{array}{l}
0 \\
1
\end{array}\right], \\
C=[1-1],
\end{gathered}
$$

and the set of state constraints $W x(k) \leq \zeta$ with

$$
W=\left[\begin{array}{cc}
0.2 & 0.2 \\
-1 & -1 \\
-1 & 0.35 \\
0.25 & -0.5 \\
0.6 & 0.1
\end{array}\right], \quad \zeta=\left[\begin{array}{l}
1 \\
1 \\
1 \\
1 \\
1
\end{array}\right]
$$


The computation of the supremal $(A, B)$-invariant set contained in $\Omega=R[W, \zeta]$ results in $\mathcal{C}^{\infty}(\Omega)=R[V, \nu]$, with:

$$
V=\left[\frac{W}{3.0857-0.7714}\right], \nu=\left[\frac{\zeta}{3.8571}\right] .
$$

The application of the algorithm presented for computation of the infimal self-bounded $(A, B)$-invariant set contained in $\Omega$ yields $\mathcal{C}_{\infty}(\Omega)=R[G, \rho]$ with:

$$
G=\left[\begin{array}{cc}
0.2 & 0.2 \\
-1 & -1 \\
-1 & 0.35 \\
0.25 & -0.5 \\
17.5 & 0 \\
-5.7143 & 0
\end{array}\right], \rho=\left[\begin{array}{c}
1 \\
1 \\
1 \\
1 \\
13.8095 \\
5.4422
\end{array}\right]
$$

The sets $\Omega, \mathcal{C}^{\infty}(\Omega), \mathcal{C}_{\infty}(\Omega)$ are shown in Figure 1 .

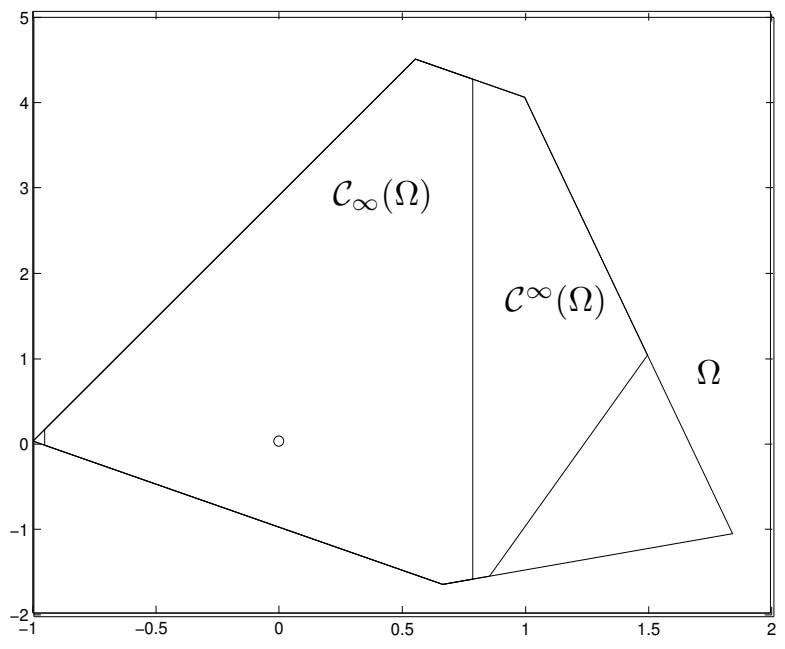

Figure 1: $\{0\} \subset \mathcal{C}_{\infty}(\Omega) \subset \mathcal{C}^{\infty}(\Omega) \subset \Omega$.

The computation of the set of admissible reference signals gives:

$$
\mathcal{Y}_{R}=\{r:-3.7209 \leq r \leq 0.8\} .
$$

For $r=0.5$, the corresponding $\bar{x}$ and $\bar{u}$ are:

$$
\bar{x}=\left[\begin{array}{l}
-0.0625 \\
-0.5625
\end{array}\right], \bar{u}=-1.0375 .
$$

It turns out that, in this example, closed-loop positive invariance of $\mathcal{C}_{\infty}(\Omega)$ can be achieved through linear state feedback $u(k)=F x(k)$ (see in e.g. (Hennet, 1995) details on how it

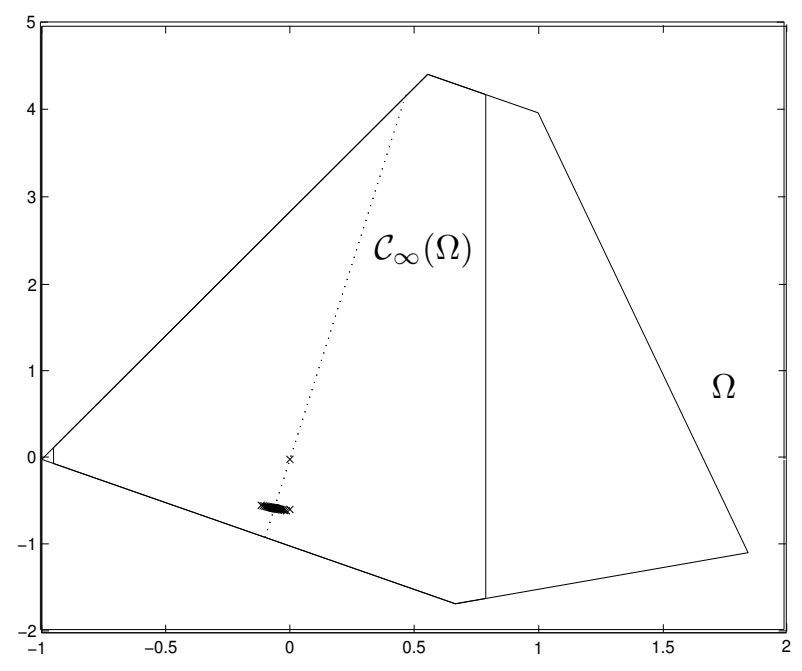

Figure 2: $\Omega, \mathcal{C}_{\infty}(\Omega)$, admissible $\bar{x}$ and the trajectory of the state.

can be checked via linear programming). Then, the application of the procedure based on variable translation, suggested in (Blanchini and Miani, 2000) for the computation of a control law such that the system can track $r=0.5$, gives the linear state feedback: $u(k)=F(x(k)-\bar{x})+\bar{u}$, with $F=\left[\begin{array}{ll}0.0079 & 0.8178\end{array}\right]$.

In Figure 2 the sets $\Omega, \mathcal{C}_{\infty}(\Omega)$ and the set of admissible $\bar{x}$ are shown, as well as a trajectory of the state under the control law computed above, with null initial state. In Figure 3 the response $y(k)$ is shown.

It can be noticed that in this example $\bar{x}$ and $\bar{u}$ are unique for the given reference $r$. However, this is not true in the general case. As suggested by one of the reviewers, this freedom on the choice of $\bar{x}$ and $\bar{u}$ could be used to optimize the computation of the tracking control law, in the sense of achieving other control goals.

\section{CONCLUSIONS}

This work presented the concept of self-bounded $(A, B)$ invariant sets of the state space for discrete-time systems. The existence of such sets implies limitations in the controllability of trajectories confined in a given set. Self-bounded polyhedral sets were geometrically and analytically characterized.

Given a convex region, there exists a self-bounded set which is contained in any other self-bounded set in this region. If a trajectory completely contained in the region starts from this infimal set, then it will not exit it. Such an infimal set was 


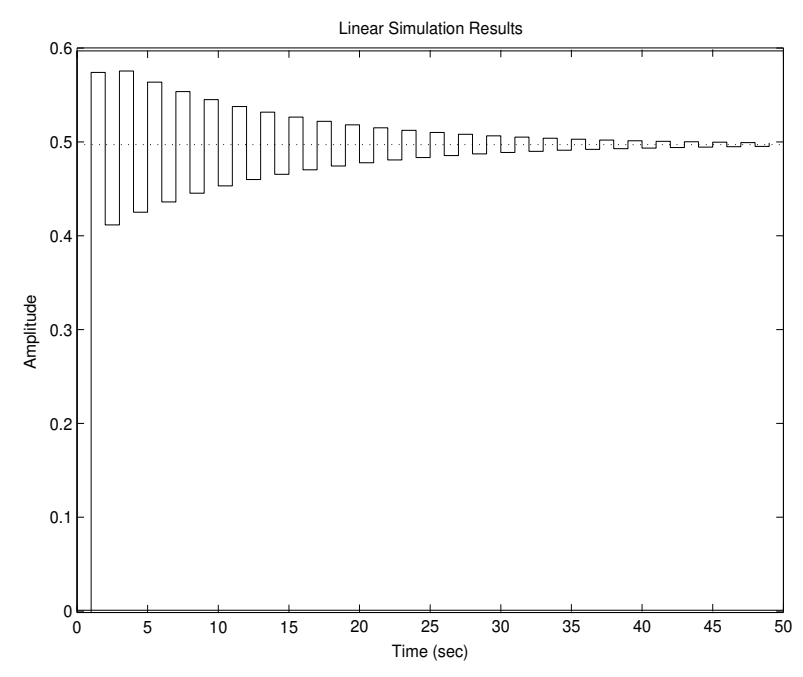

Figure 3: Time response $y(k)$.

characterized and a numerical method was presented for its computation in the polyhedral case.

Finally, it was shown how this concept can be applied in the study of a tracking problem for state constrained linear systems. The set of the constant reference signals which can be tracked was then characterized.

Although only state constraints have been treated here, as shown in (Dórea and Hennet, 2000), control constraints as well as persistent disturbances can be easily casted in the presented framework.

\section{ACKNOWLEDGEMENTS}

This work was partially supported by CNPq, Brazil, under grants \# 301439/1997-4 and 471448/01-0.

\section{REFERENCES}

Basile, G. and Marro, G. (1982). Self-bounded controlled invariant subspaces: a straightforward approach to constrained controllability, J. Optimiz. Theory Appl. 38: $71-81$.

Basile, G. and Marro, G. (1992). Controlled and Conditioned Invariants in Linear System Theory, Prentice-Hall.

Bertsekas, D. P. (1972). Infinite-time reachability of statespace regions by using feedback control, IEEE Trans. Automat. Contr. 17(5): 604-613.

Blanchini, F. (1994). Ultimate boundedness control for uncertain discrete-time systems via set-induced Lyapunov functions, IEEE Trans. Automat. Contr. 39(2): 428433.

Blanchini, F. (1999). Set invariance in control, Automatica 35(11): 1747-1767.

Blanchini, F. and Miani, S. (2000). On the tracking problem for constrained linear systems, Proc. 14th International Symposium of Mathematical Theory of Networks and Systems, Perpignan, France.

Dórea, C. E. T. and Hennet, J.-C. (1999). ( $A, B)$-invariant polyhedral sets of linear discrete-time systems, J. Optimiz. Theory Appl. 103(3): 521-542.

Dórea, C. E. T. and Hennet, J.-C. (2000). Self-bounded $(A, B)$-invariant polyhedra of discrete-time systems, Proc. 39th IEEE Conf. Decision Contr., Sydney, Australia, pp. 3163-3168.

Glover, J. D. and Schweppe, F. C. (1971). Control of linear dynamic systems with set constrained disturbances, IEEE Trans. Automat. Contr. 16(5): 411-423.

Hennet, J.-C. (1995). Discrete-time constrained linear systems, in C. Leondes (ed.), Control and Dynamic Systems, Vol. 71, Academic Press, pp. 157-213.

Keerthi, S. S. and Gilbert, E. G. (1987). Computation of minimum-time feedback control laws for discrete-time systems with state-control constraints, IEEE Trans. Automat. Contr. 32(5): 432-435.

Schrijver, A. (1987). Theory of Linear and Integer Programming, John Wiley and Sons, Chichester. 